\title{
Review Article: Time scale of the growth progress in bacterial cultures: a self-consistent choice
}

Author Name:

Alberto Schiraldi ${ }^{1}$ and Roberto Foschino

\section{Affiliation:}

Department of Food, Environmental and Nutritional Sciences (DeFENS), Università degli studi di Milano, Milan, Italy, ${ }^{1}$ Former professor of Physical Chemistry at DeFENS.

\section{Corresponding Author:}

Prof. Roberto Foschino

Citation: Schiraldi and Foschino, 2021

Time scale of the growth progress in bacterial cultures: a self-consistent choice

Received Date: 2nd September 2021

Published Date: 28th September 2021

Copyrights: Prof. Roberto Foschino

This is an open access article distributed under the Creative Commons Attribution License, which permits unrestricted use, distribution, and reproduction in any medium, provided the original work is properly cited.

\begin{abstract}
An empirical model describes the behavior of an ideal microbial culture that mimics the real one. The predicted growth curve, $\log [N(t)]$, includes the onset tail of the observed sigmoid trend, while the latency phase that precedes the cell duplication is a time gap, which does not coincide with the so-called lag phase of other models. Unsolved remains the issue of the time scale: does the origin of the time scale of the experimenter coincide with that of the microbial culture? The correlation between duration of latency phase and maximum slope of the growth trend allows determination of a "true" starting point of the growth progress as the origin of the time scale of the microbial culture. Rigid shifts in the time scale of the experimenter allow alignment of the growth trends of real microbial cultures (in excess substrate, in the same medium and at the same temperature), no matter the starting population density. The time scale of the bacterial culture allows a consistent evaluation of the extent of the latency phase that can include a "negative" time span, which is supposed to deal with some basal cell metabolism not aimed at duplication.

Some examples, dealing with real psychrotrophic bacterial cultures, support the model and perfect the prediction of the extent of the latency phase at various temperatures.
\end{abstract}

Keywords: Lag phase, microbial growth model; time scale; psychrotrophic pathogenic bacteria.

\section{Introduction about the time before cell duplication}

Since decades the predictive microbiology has helped to determine best practices of coping the risk related to the adverse effects of potential presence of pathogens and spoilage microorganisms in foods. Particularly, the early phase of the microbial growth are a major focus of interest, e.g. for the assessment of shelf-life of perishable products, as the implementation of the cold chain is definitely based on the lengthening of the latency period before the cell multiplication. Therefore, the initial point of the sigmoid growth trend of bacterial cultures has been the subject of many investigations and theoretical descriptions (Baranyi 1998, Baranyi et al. 2009, Bertrand 2019, Swinnen et al. 2004, Whiting and Bagi 2002, Yates and Smotzer 2007, Zwietering et al. 1990). The parameter proposed in most models to characterize the onset tail that precedes the exponential growth period is the socalled lag phase (or lag time), $\lambda$, that depends on the starting population density, $N_{0}$, and is inversely proportional to the maximum specific growth rate, $\mu$. With the aim to give a physiological meaning to the inception of the growth curve, $\log \left(N / N_{0}\right)$, different authors (Baranyi 1998, Baranyi et al. 2009, Bertrand 2019, Swinnen et al. 2004, Whiting and Bagi 2002, Yates and Smotzer 2007, Zwietering et al. 1990) refer to the Monod approach and/or to the Michaelis Menten kinetic model to account for the availability of critical substrates that sustain the cell duplication. Thus, these authors insert adjustment factors that are functions of the time, to modify the formal expressions of Gompertz's or logistic functions and perfect the description of the growth trend aiming at enhancing the fitness of the models. From a metabolic point of view, it is argued that, in this "waiting" time, the microorganisms would implement mechanisms of adaptation to the new environmental conditions and on varying the cell cultures, the observed differences would depend on the so-called physiological state of the starting population (Baranyi and Pin 1999, Baranyi and Pin 2001, Baranyi and Roberts 1994). Further developments of such approaches deal with the search of the single-cell lag time and the construction of the onset tail 
of the growth curve as the result of a double gamma distribution of lag times, one for the single cell and the other for the growing population (Baranyi et al. 2009). This implies introduction of new parameters and coefficients that enter the fitting treatment of the experimental data. One, therefore, needs an adequately large set of good quality experimental data to obtain reliable fits and reasonably support the assumptions of the corresponding model. Unfortunately, this is seldom the case.

Moreover, these functions leave untreated a not negligible part of the experimental evidence: the $\log \left(N / N_{0}\right) \approx 0$ data that often precede the onset tail of the sigmoid growth trend and reflect the behavior of living microbes that are not yet ready to undertake duplication. Such observation deserves the attention of the researchers interested in the applications of the predictive microbiology.

The empirical model described in previous works (Schiraldi 2017a, Schiraldi 2017b, Schiraldi 2020, Schiraldi and Foschino 2021) suggests a different approach. It views the onset tail of the growth trend as an acceleration of the growth rate; this corresponds to a decrease of the duplication time, $\tau$, which therefore may not be constant, but must change with the growth progress. When the growth rate is small, $\tau$ is large and vice versa. This also allows one to account for the decrease of the growth rate when the culture is approaching the maximum level of the population density. No physiological or bio-molecular assumptions are necessary for such a choice: it directly reflects the experimental evidence, namely, the count of the microbial colonies in a Petri dish or changes of the Optical Density in a broth culture.

The function $\tau=(\alpha / t+t / \beta)$ complies with the observed results, the parameters $\alpha$ and $\beta$ directly coming from the fit of experimental data $\left[\log \left(N / N_{0}\right) \approx 0\right.$ data are not included in the fitting treatment], and with the constraint that the growth rate, $1 / \tau$, is null for $t$ $\rightarrow 0$ and $t \rightarrow \infty$. The growth trend, $N=N_{0} 2^{t / \tau}$ for duplicating microbes, is described with a simple two-parameter function, which is consistent with the intrinsic uncertainty that usually affects the experimental data in microbiological analysis. The values of the parameters $\alpha$ and $\beta$ define the overall growth trend, including the onset tail.

Although aimed to simplify the fitting treatment of experimental data, the model corresponds to the growth progress of an ideal culture, which can be a reference standard for the real ones. The starting $N_{0}$ cells of the ideal culture are supposed to have the same age and produce $N_{0}$ synchronous generation lines without experiencing any death process.

After $\beta$ generation steps, such ideal population attains its maximum level $N_{\max }=N_{0} 2 \beta$. The predicted growth trend,

$N=N_{0} 2^{t / \tau}$ (or its logarithmic equivalent), shows an upward onset tail during which the growth pace accelerates to reach the maximum of the specific rate, $\dot{N} / N$, at $t=t^{*}=(\alpha \beta / 3)^{1 / 2}$, and progressively declines to vanish (formally, for $t \rightarrow \infty$ )

when $N=N_{0} 2^{\beta}$. Three major peculiarities of the $\log [N(\mathrm{t})]$ function are (Schiraldi 2017a, Schiraldi 2017b, Schiraldi 2020):

- at $t=t^{*}, \log \left(N^{*} / N_{0}\right)=(1 / 4) \log \left(N_{\max } / N_{0}\right)$, no matter the $\log$ base used,

- $t(0)=t^{*} / 3$, is the time span of the onset tail, defined by the $\mathrm{x}$-intercept of the straight line tangent to the $\log (N)-v s-t$ trend at $t^{*}$ (whose slope corresponds to $\mu$ when the log base is e). $t(0)$ may be identified with the so called "lag phase", $\lambda$, of most models reported in the literature,

- the y-intercept of the same straight line is $(-1 / 8) \log \left(N_{\max } /\right.$ $N_{0}$ ), no matter the log base used.
A worth noticing peculiarity of the model is that it allows one to gather different growth trends in a single master plot of reduced variables, $\xi\left(\mathrm{t}_{\mathrm{R}}\right)$ and $t_{\mathrm{R}}$, where $t_{\mathrm{R}}=t / t^{*}$ and $\xi\left(t_{\mathrm{R}}\right)=t_{\mathrm{R}}{ }^{2} /\left(3+t_{\mathrm{R}}{ }^{2}\right)$.

The experimental evidence can indicate a very long (in some cases several hours) or a practically negligible no-growth phase, namely, $\log \left(N / N_{0}\right) \approx 0$ data, before the onset of the growth curve. The model, which strictly applies to actively growing cultures (i.e., $\log \left(N / N_{0}\right)>0$ ), formally accounts for this "latency phase" with a time shift, $t_{0}$, which defines a neat separation between the time span, during which the expected sigmoid $\log (N)-v s-t$ trend is observed, and a previous no-growth period. When this is the case, $\left(t-t_{0}\right)$ replaces $t$ in the above expression for $\tau$ (and related quantities, see above), so adding a third adjustable parameter in the fitting treatment of the experimental data. Since the latency period, $t_{0}$, precedes the onset tail of the growth curve, it does not correspond to $\lambda$. For $t=t_{0}$, the straight line corresponding to the maximum slope of the growth curve crosses the level

$\left[\log \left(N_{0}\right)-1 / 8 \log \left(N_{\max } / N_{0}\right)\right]$.

Previous works (Schiraldi 2017a, Schiraldi 2017b, Schiraldi 2020, Schiraldi and Foschino 2021) demonstrate, as a simple geometric consequence, the link between $t_{0}, \log \left(N_{0}\right)$ and the parameter $\beta=\log _{2}\left(N_{\max } / N_{0}\right)$ : this implies that the latency period too is involved in the evolution of the system. For a suitable physiological interpretation, some recent papers (Atolia et al. 2020, Basan et al. 2020, Bertrand 2019, Sekar et al. 2020) could be of help.

This approach therefore extends the interest from $\lambda$ to $t_{0}$, but leaves unsolved another major issue: the potential mismatch between the time scale of the experimenter and the time scale of the microbial culture. Overlooking this crucial choice unfortunately is the usual behavior (even in the case of a long latency phase): the time origin normally is just the start of the experiment, namely, the zero point in the time scale of the experimenter, although this may not correspond to the start of the activity by the microbial cells.

The present paper tries to shed some light on this issue through some examples and a rationale to define guideline criteria.

\section{Defining the time origin of bacterial culture}

According to the Schiraldi's model, the slope of the straight line tangent to the $\log _{\mathrm{e}}(N)-v s-t$ trend at $t^{*}$ is:

$$
\left(\frac{\dot{N}}{N}\right)_{t=t^{*}}=\log _{e}(2) \frac{3 \sqrt{3}}{8} \sqrt{\frac{\beta}{\alpha}}
$$

Such slope reflects the duplicating pace of the batch culture when it reaches its maximum specific growth rate, which corresponds to the nominally "balanced growth" condition: all the catabolic and anabolic processes that underlie the cell duplication progress with the same pace (Schaechter 2006). Such condition is peculiar for a given bacterial strain in a given environment (available substrates, medium volume, temperature, water activity, $\mathrm{pH}, \mathrm{Eh}$, etc.) and does not depend on the time origin. The model predicts that the time required to attain this condition, $t^{*}$, is related to the growth parameters $\alpha$ and $\beta$, and that, at $t=t^{*}$, the bacterial population reaches $1 / 4$ of the $\log \left(N_{\max } / N_{0}\right)$ gap, no matter the $\log$ base used and the time origin.

This means that any rigid backward and forward shift of the growth curve along the time axis does not affect such constraints, leaving the choice of the time origin, $t_{\text {start }}$, substantially ambiguous.

To overcome the ambiguity of the time origin, one can take advantage from some algebraic constraints that directly come from the model (Schiraldi 2017a, 2017b): 
i. $t^{*}$ does not depend on the log base used to plot the growth curve;

ii. $\log \left(N^{*} / N_{0}\right)=(1 / 4) \log \left(N_{\max } / N_{0}\right)$, no matter the log base used; iii. the straight line tangent at $t^{*}$ crosses the $\log \left(N_{\max }\right)$ level at $t_{\text {end }}$ $=\left[t_{0}+3\left(t^{*}-t_{0}\right)\right]$

iv. $\log \left(N / N_{0}\right)_{\mathrm{t}=\text { tend }}=(3 / 4) \log \left(N_{\max } / N_{0}\right)$, no matter the log base used;

v. $t(0)=\left[t_{0}+\left(t^{*}-t_{0}\right) / 3\right]$, no matter the log base used;

vi. $\log (\mathrm{N}=1)=0$, no matter the $\log$ base used.

The easiest visualization of these constraints comes from the comparison of plots of the growth curve in different log bases. Such plots differ only for the scale of the $\log (N)$ axis, while they have the same time scale. The straight lines tangent to each trend at $t^{*}$ have different slopes (Figure 1).

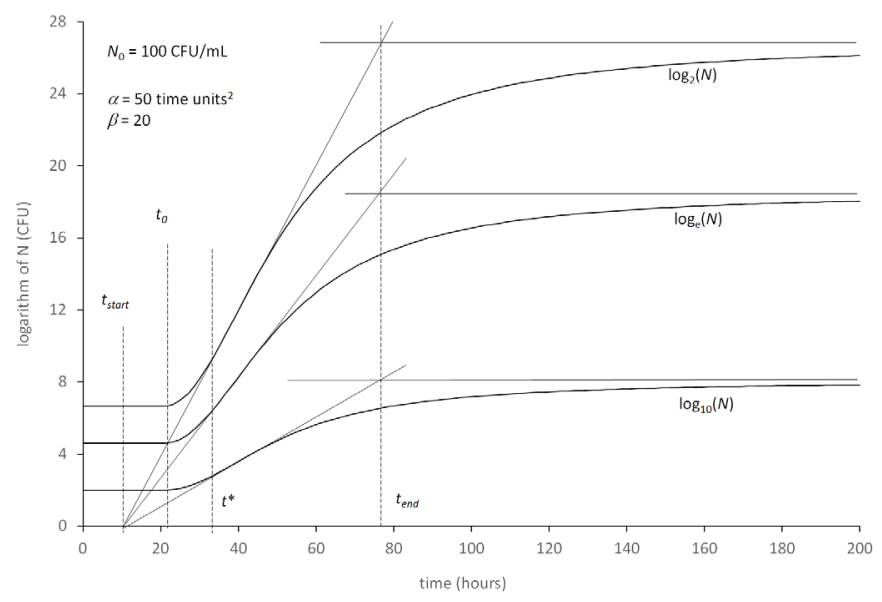

Figure 1. The same growth curve represented in different log base units.

All the straight lines tangent to the growth curves at $t=t^{*}$ must pass through the zero level, $\log (N)=0$, which corresponds to $N=1 \mathrm{CFU} / \mathrm{mL}$, no matter the $\log$ base considered. This is tantamount to say that all these straight lines must converge in the same point of the time scale, no matter the log base. The use of various log bases is just a tool for a more accurate identification of this time value, $t_{\text {start }}$, as the virtual time origin for the considered culture, which depends on the actual growth trend and not on the arbitrary choice of the experimenter. It may fortuitously coincide with the start time of the experiment, but may also follow or precede it. Accordingly, the $\left[t(0)-t_{\text {start }}\right]$ span is the time required to reach the $\mathrm{N} 0$ density starting from $N=1$ $\mathrm{CFU} / \mathrm{mL}$ with the maximum specific growth rate in the actual environmental conditions.

This choice of $t_{\text {start }}$ overcomes the ambiguity of selecting the moment when the bacterial culture really starts its activity, even if its population may be not yet increasing. For the sake of clarity, the symbol $\theta$ will represent time in the scale of the bacterial culture: $\theta=0$ therefore corresponds to $t=t_{\text {start }}$ in the time scale of the experimenter. The new scale allows a better comparison of the growth parameters, namely, $t_{0}, t(0), t^{*}$ and $t_{\text {end }}$, which becomes $\theta_{0}, \theta(0), \theta^{*}$ and $\theta_{\text {end }}$, respectively. It may happen that some $\log \left(N / N_{0}\right)=0$ data are observed for $t<t_{\text {start }}$, i.e., for $\theta<0$. They would reflect a resting metabolic state not aimed at the cell duplication and/or at the increase of cell mass.

Two extreme situations, met in a previous work (De Silvestri et al. 2018, Schiraldi and Foschino 2021), represent suitable case studies.

\subsection{Some case studies}

The reader can find experimental details in De Silvestri et al. (2018). The software Table Curve 2D (Jandel Scientific, Systat
Software Inc., San Jose, U.S.A.) has been used to draw the curves and has provided the best fit parameters.

The first case study deals with a psychrotrophic bacterium, Aeromonas hydrophila subsp. hydrophila DSM-30187 strain, cultivated at $4^{\circ} \mathrm{C}$ starting from $N_{0}=10^{6} \mathrm{CFU} / \mathrm{mL}$ (De Silvestri et al. 2018). A particularly long latency phase (about $150 \mathrm{~h}$ ) precedes the onset of the expected sigmoid trend of the growth curve. When the experimental data are reported in three different $\log$ bases (2, e and 10), the observed trends are those shown in Figure 2 (panel A), where the axis of the time is in the scale of the experimenter.
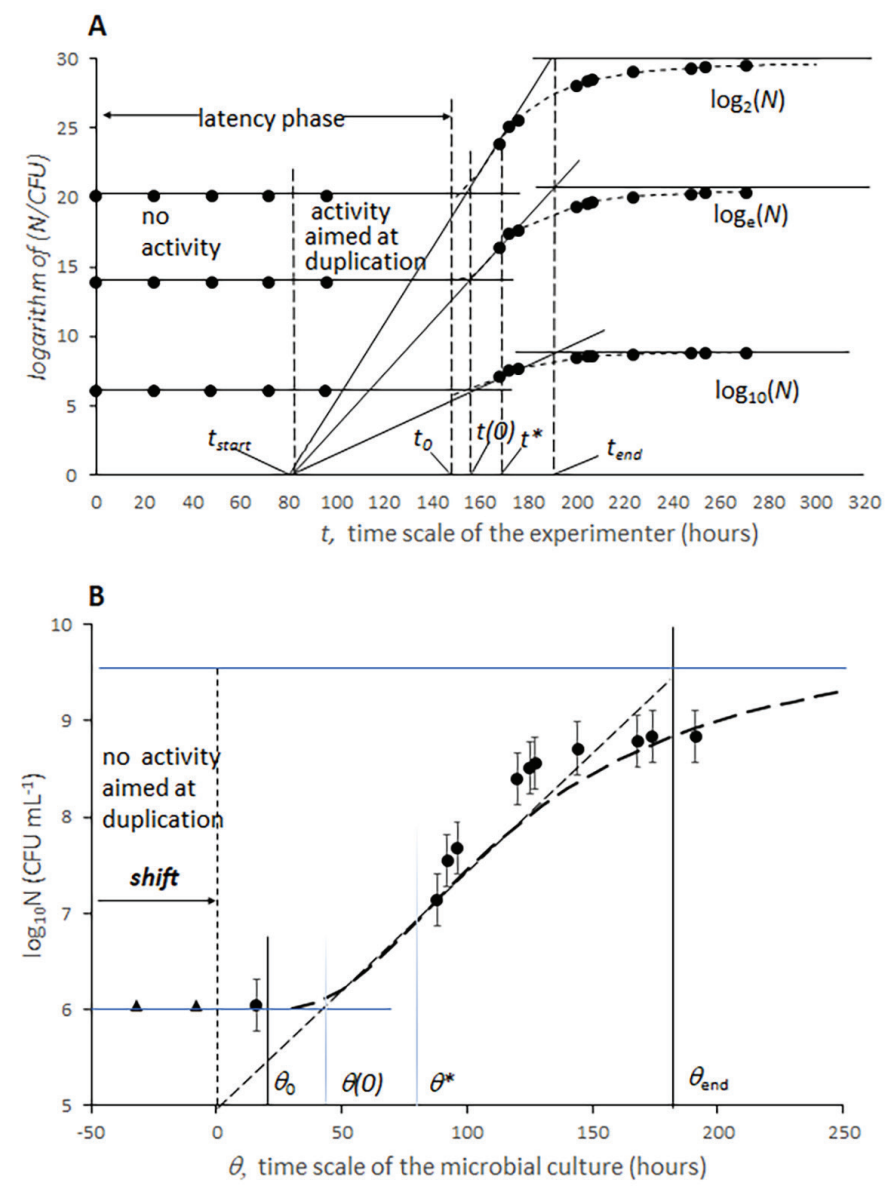

Figure 2. Growth of Aeromonas hydrophila at $4{ }^{\circ} \mathrm{C}$ starting from $N_{0}=$ $106 \mathrm{CFU} / \mathrm{mL}$. Panel A: The three curves correspond to different log base units. Panel B: Fitting adjustment of the same data. The triangles correspond to data collected during the silent inactive phase that precedes the preliminary active phase before the onset of the growth curve.

A tentative fitting treatment of $\log \left(N / N_{0}\right)>0$ data gives a rough estimation of the value of $t^{*}$ and the corresponding slope of the growth curve. Applying the above mentioned constraints (i - vi), one can identify the best convergence point for the three straight-lines tangent to the growth curve at $t^{*}$, namely, $t_{\text {start }}=80 \mathrm{~h}$ in the time scale of the experimenter. The latency period therefore encompasses a silent state for $t<t_{\text {start }}$ (about $80 \mathrm{~h}$ ), where no biological activity is aimed at cell duplication or increase of cell mass, and a pre-growth phase between $t_{\text {start }}$ and $t_{0}$, spent by the culture to adjust its biochemical panoply to undertake the onset of the growth steps, although no increase of $\mathrm{N}$ is yet observed. This procedure finally allows estimation of the relevant intercepts with the level $N_{0}$ and $N_{\text {max }}, t(0)$ and $t_{\text {end }}$, respectively and $t_{0}$.

Shifting the time scale by $80 \mathrm{~h}$ and neglecting the data collected 
during this period (namely, $\log \left(N / N_{0}\right)=0$ ), one may define the origin of the new time scale, $\theta=0$, and use the rest of the data to refine the best-fit treatment, leaving $\alpha, \beta$ and $\theta_{0}$ as fitting parameters (Figure 2, panel B).

The value of $\theta^{*}$ is $\theta^{*}=\theta_{0}+(\alpha \beta / 3)^{1 / 2}=86.8 \mathrm{~h}$ in the time scale of the microbial culture, i.e., $t^{*}=166.8 \mathrm{~h}$ in the time scale of the experimenter. At $\theta=\theta_{0}$, the straight line tangent to the growth curve at $\theta^{*}$ crosses the level $\left[\log \left(N_{0}\right)-1 / 8 \log \left(N_{\max } / N_{0}\right)\right]$. The values of the best-fitting parameters are: $\theta_{0}=22.4 \mathrm{~h}$ in the time scale of the microbial culture; $\alpha=686.3 \mathrm{~h} 2, \beta=12.9, \mathrm{R}^{2}=0.967$, $\mathrm{SE}=0^{2}$.

The second case study deals with the culture of Yersinia enterocolitica subsp. enterocolitica DSM-27689 (serotype 8 biovar 1) (De Silvestri et al. 2018), the growth curve of which

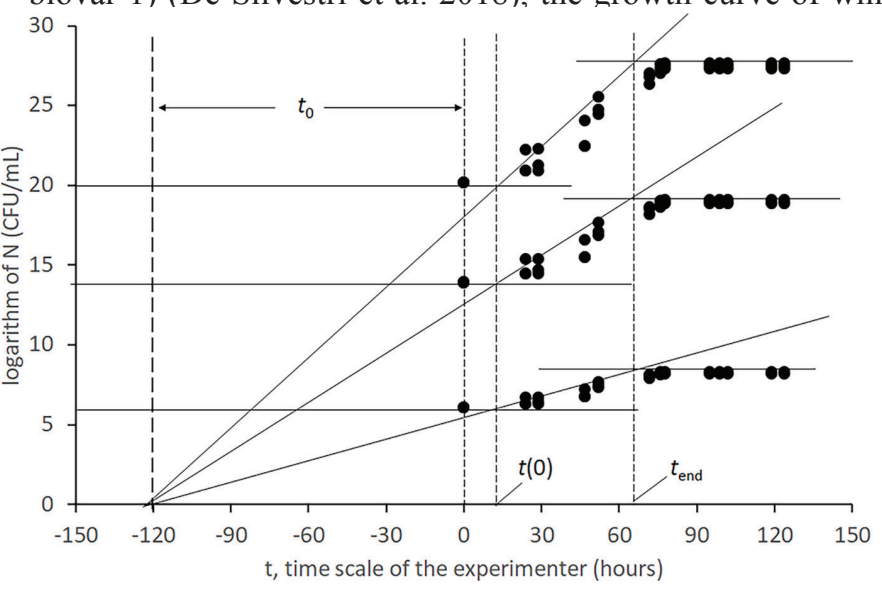

Figure 3. Growth of Yersinia enterocolitica at $4^{\circ} \mathrm{C}$ starting from $N_{0}=$ $106 \mathrm{CFU} / \mathrm{mL}$. The three curves correspond to different log bases unit).

This situation is very common, although unambiguously explained. One may venture the conclusion that the investigated culture is immediately ready to grow once transferred into the medium of the experiment (Schiraldi and Foschino 2021). A more convincing view is that the culture is not really at rest when the experiment starts. In other words, the culture has already experienced a congruous pre-growth phase before the start of the experiment: the true $t_{\text {start }}$ is "negative" with respect to the zero point of the experimenter.

In this case the straight lines tangent to the growth curve at $t^{*}$ converge for $\log (N)=0$ at $t_{\text {start }}=-120 \mathrm{~h}$ in the time scale of the experimenter, namely much before the onset of the growth progress. The fortuitous coincidence of $t_{0}$ with the zero of the time scale of the experimenter gives the fake impression of a no pre-growth phase.

\section{Looking for a general rationale}

As mentioned before, the "balanced growth" state is a characteristic of the overall system, namely, the microorganism and the closed environment in a continuous but bound changing of conditions due to the cell activities (Schaechter 2006). One therefore may assume that the corresponding maximum specific growth rate, $(\dot{N} / N)^{*}$, and the maximum sustainable population, $N_{\max }$, in given growth conditions, are intrinsic properties of the ideal batch culture proposed in the Schiraldi's model.

In the time scale of the experimenter (and $\log _{2}$ scale for $\mathrm{N}$ ), the expression of the steepest straight-line tangent to the growth curve is (see "supporting information" in (Schiraldi and Foschino 2021):

$\log _{2}(N)=\frac{3 \sqrt{3}}{8} \sqrt{\frac{\beta}{\alpha}} t-\left[\frac{\beta}{8}+\frac{3 \sqrt{3}}{8} \sqrt{\frac{\beta}{\alpha}} t_{0}-\log _{2}\left(N_{0}\right)\right]$

The value of $t_{\text {start }}$ is identified putting the condition $\log _{2}(N)=0$, namely,

$$
t_{\text {start }}=\frac{\frac{\beta}{8}+\frac{3 \sqrt{3}}{8} \sqrt{\frac{\beta}{\alpha}} t_{0}-\log _{2}\left(N_{0}\right)}{\frac{3 \sqrt{3}}{8} \sqrt{\frac{\beta}{\alpha}}}=t_{0}-\frac{\log _{2}\left(N_{0}\right)-\frac{\beta}{8}}{\frac{3 \sqrt{3}}{8} \sqrt{\frac{\beta}{\alpha}}}
$$

Reminding that $(3 \sqrt{ } 3) / 8 \sqrt{ }(\beta / \alpha)$ is the "slope" of the straight line, the duration of the latency phase in the time scale of the microbial culture is:

$$
\begin{aligned}
\left(t_{0}-t_{\text {start }}\right)=\theta_{0}= & \frac{\log _{2}\left(N_{0}\right)-\frac{\beta}{8}}{\text { slope }}=\frac{\log _{10}\left(N_{0}\right)-\frac{\beta}{8} \log _{10}(2)}{\log _{10}(2) \text { slope }} \\
& =\frac{9 \log _{10}\left(N_{0}\right)-\log _{10}\left(N_{\max }\right)}{\log _{10}(2) \text { slope }} \lessgtr 0
\end{aligned}
$$

$\theta<\theta_{0}$ values correspond to a latency phase (or a part of it, see Figure 2) during which the cell activity simply sustains a basal metabolism that allows survival but not preparation to duplication.

The experimental evidence (Schiraldi and Foschino 2021) suggests that growth curves of a given microbial culture starting from different $N_{0}$ levels, but incubated at the same temperature, have approximately the same maximum specific growth rate, namely, the same steepest slope. They could align on the same straight line starting from a common origin by rigid shifts along the time scale of the experimenter, no matter the shift direction and the selection of the common $t_{\text {start }}$. Such alignment allows a better view of the different $\theta_{0}$ values.

Taking $t_{\text {start }}$ as the "true" time origin, one may define a $\log (N)-$ $v s-\theta$ plot to draw a straight line starting from the origin with the same slope as the tangent to the growth curve, $\log [N(\theta)]$, at $\theta^{*}$. This straight line crosses the level $\log \left(N_{\max }\right)$ at $\theta=\theta_{\text {end }}$. Slope of the straight line and $N_{\max }$ are empirical parameters, determined through the best-fit treatment of experimental data: here they refer to as intrinsic peculiarities of the closed system, bacterial population and environmental conditions.

With such premises, it is soon apparent that the $N_{0}$ value will govern the position of the $y$-axis, since, according to constraints of the model, the $y$-intercept of the straight line is $-1 / 8 \log \left(N_{\max } /\right.$ $N_{0}$ ), no matter the log base used. This leads to identify:

- $\theta_{0}$ as the upper limit of the no-growth gap between $\mathrm{t}_{\text {start }}$ and the onset of the growth curve, and

- $\theta^{*}$, since $\left(\theta_{\text {end }}-\theta_{0}\right)=3\left(\theta^{*}-\theta_{0}\right)$, according to the Schiraldi's model (Schiraldi 2017a, Schiraldi 2017b, Schiraldi 2020, Schiraldi and Foschino 2021). 
Figure 4 reports this simulation for three different $N_{0}$ values in the $\log _{10}$ units.

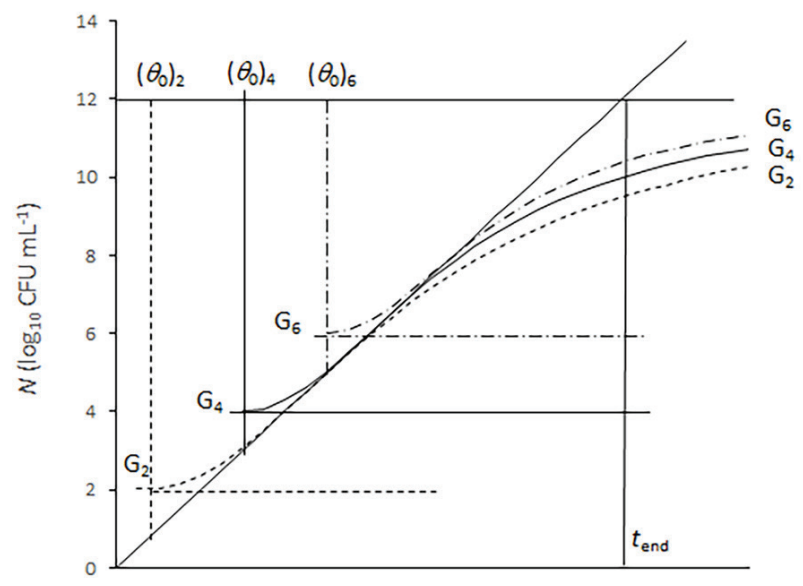

$\theta$, time scale of the microbial culture (a.u.)

Figure 4. Growth curves of virtual cultures with the same maximum specific growth rate and maximum sustainable population density, $N_{\max }$ starting from different population density, $N_{0}$ : subscripts reflect the $\log _{10}\left(N_{0}\right)$ values.

Equation (4) indicates that $\theta_{0}$ is null when $\log _{2}\left(N_{0}\right)=-\beta / 8$, and linearly increases with increasing $\log \left(N_{0}\right)$.

One can soon verify that, for a given $\log \left(N_{\max }\right), \theta_{0}$ is proportional to $\log \left(N_{0}\right)$. It looks like if the cells may require a longer time to adjust their biochemical machinery to comply the "balanced growth" condition when their starting population is larger: the smaller the $\log \left(N_{\max } / N_{0}\right)$ gap, the larger the reluctance to undertake the growth steps.

Thus, a specific procedure to fit the growth curve may be defined as follows:

a) determine the experimental data and give them the $\log (N)$ format (no matter the log base);

b) use only data relevant to the progress of the growth (no $\log (N /$ $N_{0}$ ) $=0$ data) for the fitting treatment;

c) calculate the straight line tangent to the growth curve at the maximum slope point in the $\log (N)-v s-t$ plot;

d) calculate the $\mathrm{x}$-intercept of this straight line at the level $\log (N)$ $=0$. This identifies $t_{\text {start }}$;

e) determine the time gap between the start of the experiment and $t_{\text {start }}$ is the shift (positive or negative) to apply;

f) adjust the time scale taking $t_{\text {start }}$ as the time origin;

g) determine the intercepts of the straight line with the levels $\log (N)=\log \left(N_{0}\right), \log (N)=\log \left(N_{\max }\right)$ and $\log (N)=\left[\log \left(N_{0}\right)-\right.$ $\left.\left.1 / 8 \log \left(N_{\max }\right) / N_{0}\right)\right]$ : these intercepts occur at $\theta(0), \theta_{\text {end }}$ and $\theta_{0}$, respectively.

A couple of case studies (data from De Silvestri et al. 2018) can exemplify the above rationale.

Two Yersinia enterocolitica cultures starting from different $N_{0}$ densities $\left(10\right.$ and $\left.10^{6} \mathrm{CFU} / \mathrm{mL}\right)$ in the same medium and at the same temperature $\left(4^{\circ} \mathrm{C}\right)$ show the growth curves reported in Figure 5 (panel A).
A

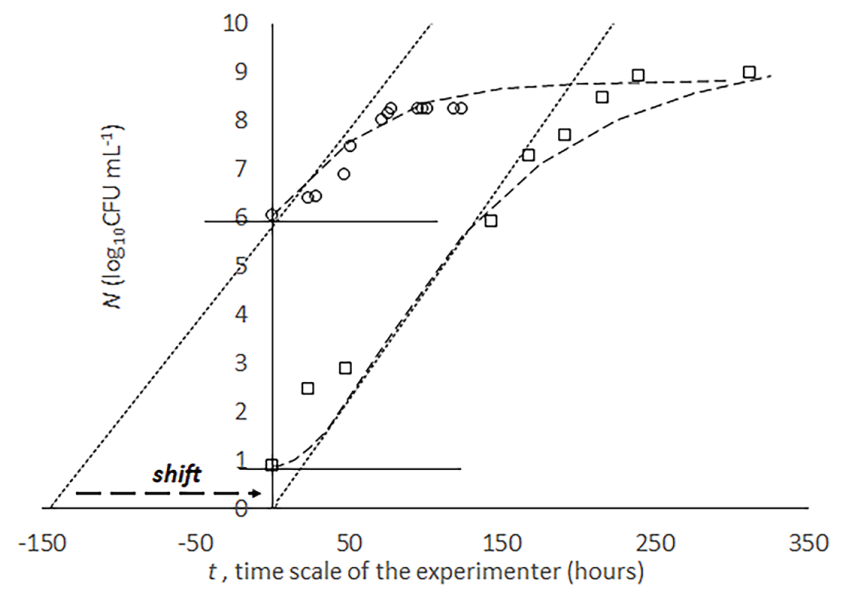

B

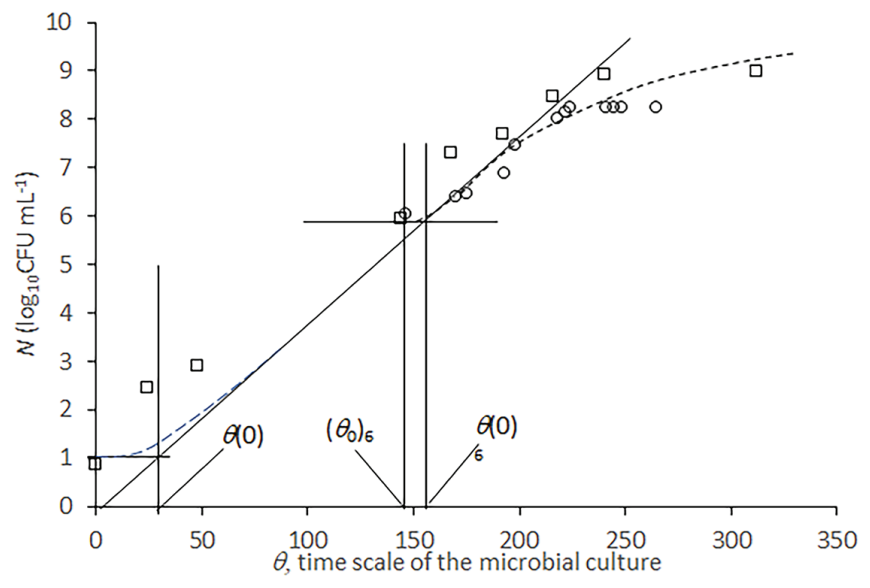

Figure 5. Panel A: growth curves of Y.enterocolitica starting from 10 and $10^{6} \mathrm{CFU} / \mathrm{mL}$ at $4^{\circ} \mathrm{C}$. Panel $\mathrm{B}$ : the same data in the time scale of the microbial culture. The subscripts 1 and 6 correspond to the $\log _{10}\left(N_{0}\right)$ values.

These data show close slopes and $N_{\max }$ levels, but the two parallel straight lines corresponding to the maximum of the specific growth rate have different $\mathrm{x}$-intercepts. The search for a common origin therefore requires a rigid shift of either growth curve to achieve a full alignment of the two trends.

One can define a Cartesian $\log _{10}(N)-v s-\theta$ plot where a straight line starting from the origin crosses the estimated common horizontal level, $N_{\max }$, with a slope that corresponds to the maximum growth rate suggested by the experimental evidence. The time of the cross point at $\log \left(N_{\text {max }}\right)$ is $\theta_{\text {end }}$ on the time scale of the bacterial culture. The same straight-line crosses each starting population level (10 and $\left.10^{6} \mathrm{CFU} / \mathrm{mL}\right)$ at the respective $\theta(0)$ points. The comparison between these values and those on the time scale of the experimenter suggests the width and the direction of the rigid shift for each growth curve: 4 and $146 \mathrm{~h}$ for $N_{0}=10$ and $10^{6} \mathrm{CFU} / \mathrm{mL}$, respectively. The alignment produces the plot in Figure 5 (panel B).

Finally, applying the above treatment steps f) and g), one can determine the relevant values of $\theta(0)$ and $\theta_{0}$. In the present case, $\left(\theta_{0}\right)_{1}$ coincides with the zero of either time scale (experimenter or microbes), while $\left(\theta_{0}\right)_{6}=120 \mathrm{~h}$ (to compare with $t_{0} \approx 0 \mathrm{~h}$ in the time scale of the experimenter). This analysis leads to the conclusion that the microbial population with a starting density $N_{0}=10$ $\mathrm{CFU} / \mathrm{mL}$ is actually ready to undertake the cell duplication at 
the start of the experiment, while the other culture with $N_{0}=10^{6}$ $\mathrm{CFU} / \mathrm{mL}$ has someway experienced a $120 \mathrm{~h}$ hidden, although biologically active, latency before the start of the experiment.

Similar results come from two cultures of Listeria monocytogens at $7^{\circ} \mathrm{C}$ (Figure 6) applying a -45 and $+70 \mathrm{~h}$ shift to the growth curves starting from $N_{0}=10$ and $10^{6} \mathrm{CFU} / \mathrm{mL}$, respectively. The intercepts with the level $\left[\log \left(N_{0}\right)-1 / 8 \log \left(N_{\max } / N_{0}\right)\right]$ (heavy horizontal line in the figure on the right hand side) determine the corresponding values of $\theta_{0}$.

A

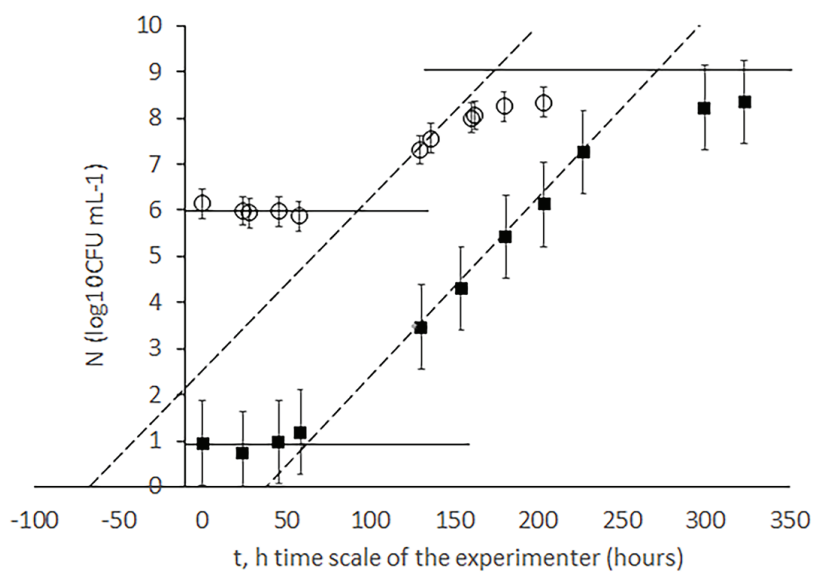

B

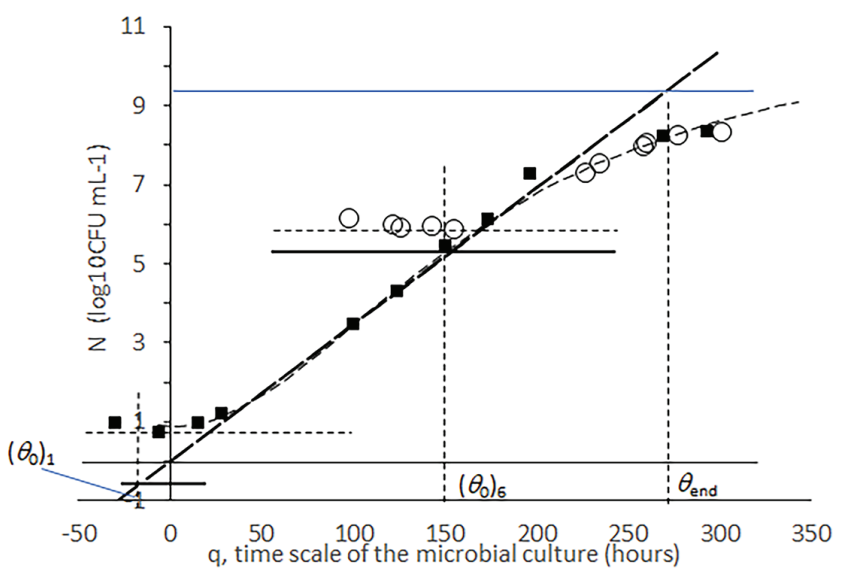

Figure 6. Panel A: growth curves of L. monocytogenes starting from 10 and $10^{6} \mathrm{CFU} / \mathrm{mL}$ at $7^{\circ} \mathrm{C}$. Panel B: the same data in the time scale of the microbial culture. The subscripts 1 and 6 correspond to the $\log _{10}\left(N_{0}\right)$ values.

The culture starting from $N_{0}=10 \mathrm{CFU} / \mathrm{mL}$ shows $\theta_{0}<0$. As a general statement, $\theta<0$ values reflect activity not directly aimed at the cell duplication. The culture starting from $N_{0}=10^{6} \mathrm{CFU} /$ $\mathrm{mL}$ shows an almost $150 \mathrm{~h}$ latency phase before the onset tail of the growth curve, which is much more than predicted in the previous work, $t_{0} \approx 40 \mathrm{~h}$ (Schiraldi and Foschino 2021).

These examples show that reference to the time scale of the microbial culture reveals unexpected traits of the latency phase that precedes the onset of the sigmoid growth trend.

\section{What happens on increasing temperature?}

If a set of experimental growth curves recorded at different temperatures is available for a given type of closed growth system (bacterial population and relevant environmental conditions), then each of them will show different values of the maximum slope in the $\log (N)-v s-t$ plot.

Once each growth curve is aligned with the straight line starting from the relevant $t_{\text {start }}$ (taken as the common time origin in the scale of the microbial culture) at the level $\log (N)=0$ with a slope indicated by the best fit of the experimental data, then the overall evidence will appear as in Figure 7. The slope of the straight line increases with increasing temperature, while the corresponding $\theta_{0}$ values decrease.

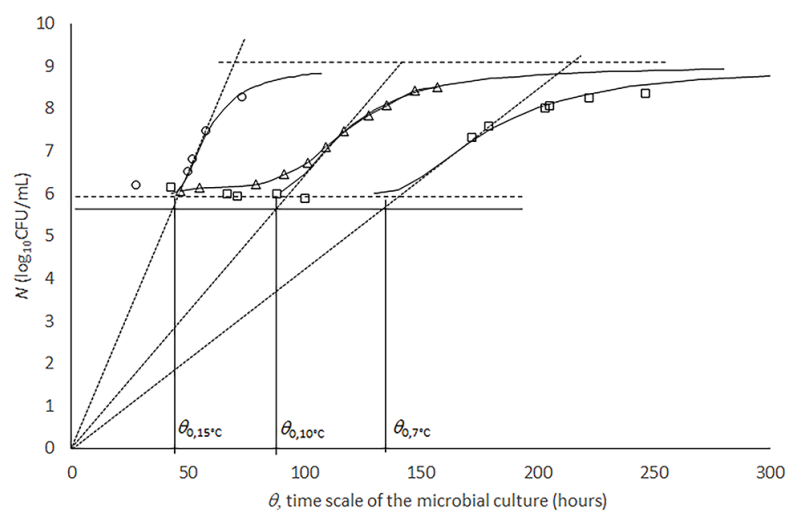

Figure 7. Scaled growth curves of L. monocytogenes from $N_{0}=10^{6} \mathrm{CFU} /$ $m L$ at 7 (squares), 10 (triangles) and $15^{\circ} \mathrm{C}$ (circles). The intercepts with the level $\left[\log \left(N_{0}\right)-1 / 8 \log \left(N_{\max } / N_{0}\right)\right]$ (heavy horizontal line) determine the corresponding values of $\theta_{0}$.

For the case reported in Figure 7 the final growth level, $N_{\max }$, is practically (within the experimental error) the same for all the growth curves considered. Differences of $N_{\max }$ values normally are small for batch cultures of a given bacterial species in a given medium; nonetheless, they would not affect the considerations reported below. The maximum slope of the growth curve for $\log _{2}(N)$ data, namely, slope $=(3 \sqrt{ } 3) / 8 \sqrt{ }(\beta / \alpha)$, leads to a simple expression for the corresponding straight line starting from the time origin in the scale of the microbial culture,

$$
y=\left[\frac{3 \sqrt{3}}{8} \sqrt{\frac{\beta}{\alpha}}\right] \theta=\text { slope } \times \theta .
$$

Since this line crosses the level $\left[\log _{2}\left(N_{0}\right)-\beta / 8\right]$ at $\theta=\theta_{0}$, one can easily obtain the corresponding expression for $\theta_{0}$ :

$$
\theta_{0}=\frac{\log _{2}\left(N_{0}\right)}{\text { slope }}-\frac{1}{8} \frac{\beta}{\text { slope }}
$$

Thence, for given slope and starting level, $N_{0}, \theta_{0}$, decreases with $\beta=\log \left(N_{\max } / N_{0}\right)$. Since the slope increases with increasing temperature, $\mathrm{T}$, the opposite occurs for $\theta_{0}$ (Figure 7 ). For given $\mathrm{T}$ and $\beta$, the duration of the latency phase is proportional to $\log \left(N_{0}\right)$. The above relationships suggest that the latency phase is related not only to the starting level, $N_{0}$, but also to the overall extent, $\beta=\log \left(N_{\max } / N_{0}\right)$, of the forthcoming growth progress. It looks like if the starting microbial population would spend the nogrowth latency period $\left(0<\theta<\theta_{0}\right)$ to "plan" its future evolution. Such conclusion is in line with recent literature reports (Bertrand 2019), once $\theta_{0}$ replaces $\lambda$ in therein discussion.

Once again, a case study can be of help to clarify the picture and highlight some important consequence of an appropriate evaluation of $\theta_{0}$.

Cultures of $Y$. enterocolitica were grown in the same medium at different temperatures $\left(4,7,10,15,25,30^{\circ} \mathrm{C}\right)(\mathrm{De}$ Silvestri et al. 2018), starting from $N_{0}=10$ and $10^{6} \mathrm{CFU} / \mathrm{mL}$. The best fit treatment of these data according to the Schiraldi's model 
leads for all of them to practically the same $N_{\max }$ values that correspond to $\beta=35$ and 10.5 , respectively, while the values of $\alpha$ strongly decrease with increasing temperature (Schiraldi and Foschino 2021). From the $\alpha$ and $\beta$ values, one can calculate the relevant $\theta_{0}$ through equation (6). It is worth noticing that such $\theta_{0}$ values strongly differ from the $t_{0}$ estimated in the previous paper (Schiraldi and Foschino 2021) in the time scale of the experimenter (i.e., $t_{0} \approx 0$ at every temperature). Figure 8 shows the $\theta_{0}-v s-T$ decreasing trends that suggests two temperature domains, below and above $15^{\circ} \mathrm{C}$, respectively.
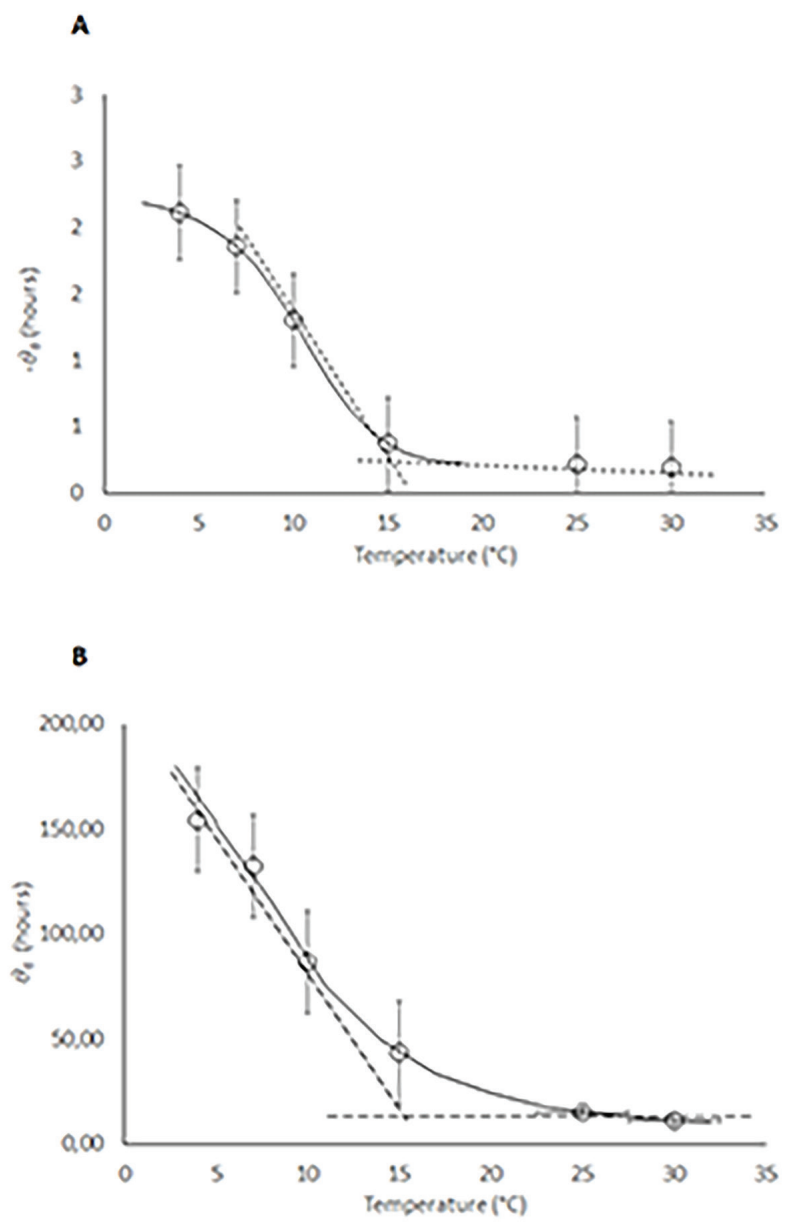

Figure 8. Trend of the duration of the latency phase, in the time scale of the microbes, for Y. enterocolitica cultures Panel $A N_{0}=10$, Panel $B N_{0}$ $=10^{6} \mathrm{CFU} / \mathrm{mL}$ ) at various temperatures.

It is worth noticing that the $\theta_{0}$ values for $N_{0}=10 \mathrm{CFU} / \mathrm{mL}$ (Figure 8, panel A) are small and negative for $\mathrm{T}<15^{\circ} \mathrm{C}$ : this means that the corresponding latency phase does not deal with the preparation of cell duplication, which actually begins for $\theta \geq \theta_{0}$. The latency phase instead has a practically negligible duration for $\mathrm{T}>15^{\circ} \mathrm{C}$.

The $\theta_{0}$ values for $N_{0}=10^{6} \mathrm{CFU} / \mathrm{mL}$ are large and positive (Figure 8 , panel $\mathrm{B})$, although becoming practically negligible for $\mathrm{T}>15^{\circ} \mathrm{C}$.

A similar picture describes the data dealing with cultures of Listeria monocytogenes (Figure 9).
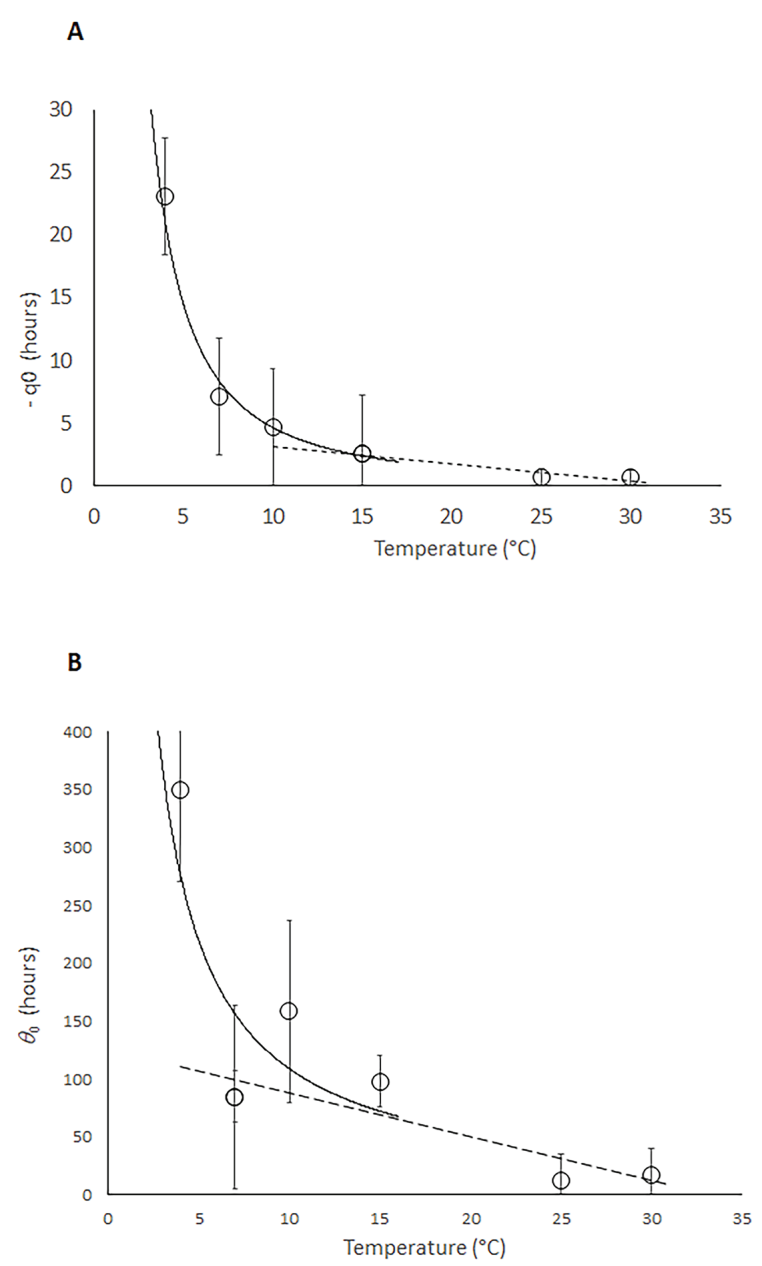

Figure 9. Trend of the duration of the latency phase, in the time scale of the microbes, of L. monocytogenes cultures (panel $A$ : $N_{0}=10$, panel B: $N_{0}=10^{6} \mathrm{CFU} / \mathrm{mL}$ ) at various temperatures.

This evaluation of $\theta_{0}$ in the time scale of the microbes confirms the expectation of a behavior change around $15^{\circ} \mathrm{C}$, determined from the values of $t^{*}$ in the scale of the experimenter (Schiraldi and Foschino 2021). The fact that both these psychrotrophic cultures show an almost null latency phase for $\mathrm{T}>15^{\circ} \mathrm{C}$, no matter the value of $N_{0}$, is of some relevance in view of the microbial spoilage of food products that can host these microbes.

\section{Conclusions}

An empirical model describes the behavior of an ideal microbial culture that mimics the real one, since its parameters come from the best fit of the experimental growth curves of duplicating microbes. However, the model leaves unsolved the issue of the time scale: does the origin of the time scale of the experimenter coincides with that of the microbial culture? The answer comes from the correlation between the duration of the latency phase and the forthcoming growth trend. Such correlation allows definition a time scale with origin at $t=t_{\text {start }}$, which is the $\mathrm{x}$-intercept (namely, for $\log (N)=0$ ) of the straight line with a slope equal to the maximum specific growth observed experimentally and tangent to the experimental growth trend.

A general rationale assumes that such $t_{\text {start }}$ is an intrinsic property of the whole closed system. This means that the growth trends of real microbial batch cultures align on the same straight line with origin in $t_{\text {start }}$, no matter the starting population density, $N_{0}$, of the experiment. To meet such condition, rigid shifts of the observed trends are necessary along the time scale of the experimenter. The new time scale, where $\theta=0$ corresponds to $t=t_{\text {start }}$, allows estimation of the corresponding duration of the latency phase, 
$\theta_{0}$ : the smaller $N_{0}$, the shorter $\theta_{0}$. Moreover, $\theta_{0}$ is related to the overall growth extent, $\log \left(N_{\max } / N_{0}\right)$, which is tantamount to say that the starting microbial population may "plan" its forthcoming evolution during the no-growth latency phase.

Some case studies dealing with real microbial cultures support this view and imply quite unexpected durations of the latency phase, which nonetheless are consistent with the other best-fit parameters of the model and their dependence on temperature.

The proposed approach provides the possibility of verifying the actual condition of the starting culture used to determine the growth curve, especially when the latency phase seems practically negligible or very long in the time scale of the experimenter. In the former case, the starting culture could be not really at rest: this obliges the experimenter to revise the experimental approach, like, for example, the protocol for inoculum preparation or dilution. In the latter case, all the $\mathrm{N}$ values collected at $\theta<\theta_{0}$ are not relevant to the cell duplication: they indicate that the starting culture actually is at rest being in a state of basal metabolism. Such condition is the most adequate for a correct experimental design and is advisable before using the fit of the growth curve for any consideration about the underlying microbial physiology.

\section{References}

Atolia, E., Cesar, S., Arjes, H. A., Rajendram, M., Shi, H., Knapp, B. D., Khare, S., Aranda-Díaz, A., Lenski, R. E., Huang, K. C. 2020. Environmental and physiological factors affecting high-throughput measurements of bacterial growth. Molecular Biol. Physiol., 11 (5), doi:10.1128/mBio.01378-20.

Baranyi, J. 1998. Comparison of stochastic and deterministic concepts of bacterial lag. J. Theor. Biol., 192, 403-408.

Baranyi, J., George, S.M., Kutalik, Z. 2009. Parameter estimation for the distribution of single cell lag times. J. Theor. Biol., 259, $24-30$.

Baranyi, J., Pin., C. 1999. Estimating bacterial growth parameters by means of detection times. App. Environ. Biol., 65, 732-736.

Baranyi, J., Pin, C. 2001. A parallel study on bacterial growth and inactivation. J. Theor. Biol., 210, 327-336.

Baranyi, J., Roberts, T. A. 1994. A dynamic approach to predicting bacterial growth in food. Int. J. Food Microbiol. 23, 277-294. doi: 10.1016/0168-1605(94)90157-0.

Basan, M., Honda, T., Christodoulou, D., Hörl. M., Chang, Y-F, Leoncini, E., Mukherjee, A., Okano, H., Taylor, B. R., Silverman, J. M., Sanchez, C., Williamson, J. R., Paulsson, J., Hwa, T. and Sauer, U. 2020. A universal trade-off between growth and lag in fluctuating environments. Nature, 584, 470-493.

Bertrand, R. L. 2019. Lag phase is a dynamic, organized, adaptive, and evolvable period that prepares bacteria for cell division. J. Bacteriol., 201 (7) 1 -21. https://doi.org/10.1128/ JB.00697-18.
De Silvestri, A., Ferrari, E., Gozzi, S., Marchi, F. and Foschino, R. 2018. Determination of temperature dependent growth parameters in psychrotrophic pathogen bacteria and tentative use of Mean Kinetic Temperature for the microbiological control of food. Front Microbiol 9, 3023. https://doi.org/10.3389/ fmicb.2018.03023.

Kutalik, Z., Razaz, M., Baranyi, J. 2005. Connection between stochastic and deterministic modelling of microbial growth. J. Theor. Biol., 232, 285-299.

Schaechter, M. 2006. From growth physiology to systems biology. Int. Microbiol.,9, no. 3, pp. 157-161.

Schiraldi, A. 2017a. Microbial growth in planktonic conditions. Cell Develop. Biol., 6, 1-6. doi: 10.4172/2168- 9296.1000185.

Sekar, K., Linker, S. M., Nguyen, J., Grünhagen, A., Stocker, R., Sauer, U. 2020. Bacterial glycogen provides short-term benefits in changing environments. App. Environ. Microbiol. 86 (9) e00049-20.

Swinnen, I.A.M., Bernaerts, K., Dens, E.J.J., Geeraerd, A.H., Van Impe, J.F. 2004. Predictive modelling of the microbial lag phase: a review. Int. J. Food Microbiol., 94, 137 - 159.

Schiraldi, A. 2017b. A self-consistent approach to the lag phase of planktonic microbial cultures. Single Cell Biol 2017, 6, 3 . doi: 10.4172/2168-9431.1000166.

Schiraldi, A. 2020. Growth and decay of a planktonic microbial culture. Int. J. Microbiology, 2020, Article ID 4186468, doi.10.1155/2020/4186468.

Schiraldi, A., Foschino, R. 2021. An alternative model to infer the growth of psychrotrophic pathogenic bacteria. J. Appl. Microbiol., doi: 10.1111/JAM.15215 and related "Supporting Information".

Whiting, R.C., Bagi, L.K. 2002. Modeling the lag phase of Listeria monocytogenes. Int. J. Food Microbiol., 73, 291- 295.

Yates, G. T. and Smotzer, T. 2007. On the lag phase and initial decline of microbial growth curves. J. Theor. Biol., 244, 511517.

Zwietering, M. H., Jongenburger I., Rombouts F. M., van't Riet, K. 1990. Modeling of the bacterial growth curve. Appl. Environ. Microbiol., 56, 1875-1881. 